\title{
Neutrino-antineutrino oscillations induced by strong magnetic fields in dense matter
}

\author{
Hirokazu Sasaki॰* and Tomoya Takiwaki® ${ }^{\dagger}$ \\ Division of Science, National Astronomical Observatory of Japan, 2-21-1 Osawa, \\ Mitaka, Tokyo 181-8588, Japan
}

(Received 7 June 2021; accepted 18 June 2021; published 16 July 2021)

\begin{abstract}
We simulate neutrino-antineutrino oscillations caused by strong magnetic fields in dense matter. With the strong magnetic fields and large neutrino magnetic moments, Majorana neutrinos can reach flavor equilibrium. We find that the flavor equilibration of neutrino-antineutrino oscillations are sensitive to the values of the baryon density and the electron fraction inside the matter. The neutrino-antineutrino oscillations are suppressed in the case of the large baryon density in neutron- (proton)-rich matter. On the other hand, the flavor equilibration occurs when the electron fraction is close to 0.5 even in the large baryon density. From the simulations, we propose a necessary condition for the equilibration of neutrinoantineutrino oscillations in dense matter. We also study whether such a necessary condition is satisfied near the protoneutron star by using results of neutrino hydrodynamic simulations of core-collapse supernovae. In our explosion model, the flavor equilibration would be possible if the magnetic field on the surface of the protoneutron star were larger than $10^{14} \mathrm{G}$, which is the typical value of the magnetic fields of magnetars.
\end{abstract}

DOI: 10.1103/PhysRevD.104.023018

\section{INTRODUCTION}

Neutrinos are produced through weak interactions in various explosive astrophysical sites [1]. The detection of neutrino bursts (approximately 20 events) from Supernova 1987A has opened up the possibility of identifying explosive dynamics and properties of particle physics from neutrino observations [2-4]. Current operational neutrino observatories can detect approximately $10^{4}-10^{6}$ events during a neutrino burst from a supernova in our galaxy (see, e.g., reviews in [5-12]). High statistical neutrino signals in neutrino detectors help investigate the detailed mechanism of the explosion and behaviors of neutrino oscillations inside the progenitor star.

Neutrinos propagating inside astrophysical sites are affected by neutrino coherent forward scatterings with background particles. Charged current interactions of $\nu_{e}\left(\bar{\nu}_{e}\right)$ with background electrons induce significant flavor conversions called the "Mikheyev-Smirnov-Wolfenstein (MSW) effect" $[13,14]$ when the number density of electrons inside the star decreases down to a critical density. Neutrino-neutrino interactions in dense neutrino gas cause a self-refraction

\footnotetext{
"hsasaki@lanl.gov

takiwaki.tomoya.astro@gmail.com
}

Published by the American Physical Society under the terms of the Creative Commons Attribution 4.0 International license. Further distribution of this work must maintain attribution to the author(s) and the published article's title, journal citation, and DOI. Funded by SCOAP. term in the neutrino Hamiltonian [15-28]. It is believed that, in core-collapse supernovae (CCSNe), the nonlinear potential of the self-refraction effect induces "collective neutrino oscillations" (CNOs) outside a protoneutron star (PNS) (see, e.g., a review in Ref. [29]). Earlier numerical studies of CNO [30-39] found the so-called spectral splits/ swap phenomenon, which exchanges neutrino spectra around certain critical energies. In the last decade, "multiangle calculations" were carried out by employing results of neutrino radiation hydrodynamics of CCSNe [40-49]. In such multiangle simulations, the $\mathrm{CNO}$ increases energetic $\nu_{e}$ and $\bar{\nu}_{e}$ even though the "matter suppression" $[39,43,50,51]$ smears the spectral splits in the neutrino distribution. The enhanced spectra of $\nu_{e}$ and $\bar{\nu}_{e}$ potentially affect neutrino detections [44,47-49] and nucleosynthesis inside the star such as the $\nu p$ process [45,52-54] and $\nu$ process [55]. In neutron star mergers, neutrino-neutrino interactions can induce the "matter neutrino resonances" [56-64] and affect rapid neutron capture process ( $r$-process) nucleosynthesis $[57,65,66]$. Furthermore, the possibility of fast-pairwise collective neutrino oscillations which may occur in the scale of approximately $\mathcal{O}\left(10^{-5}\right) \mathrm{km}$ are studied in CCSNe, neutron star mergers, and the early Universe (see, e.g., a review in Ref. [67]).

The finite neutrino magnetic moment can lead to flavor conversions between left-handed neutrinos and righthanded (anti)neutrinos in strong magnetic fields. Such a magnetic field effect results in conversions between active and sterile neutrinos in the case of Dirac neutrinos and neutrino-antineutrino oscillations in the case of Majorana 
neutrinos. The neutrino magnetic moment is a key to investigating new physics beyond the standard model (see, e.g., reviews in Refs. $[68,69])$. The value of neutrino magnetic moment is constrained from recent neutrino experiments, e.g., GEMMA [70], Borexino [71], XMASS-I [72], and XENON1T [73]. Among such neutrino experiments, the Borexino experiment provides a stringent upper limit on the neutrino magnetic moment: $\mu_{\nu}<2.8 \times 10^{-11} \mu_{B}$ (90\% C.L.) [71], where $\mu_{B}$ is the Bohr magneton. The value of the neutrino magnetic moment can be also constrained from neutrino energy loss in globular clusters $[74,75]$ and intermediate-mass stars [76]. Currently, the most stringent upper limit on the neutrino magnetic moment is $\mu_{\nu}<$ $1.2 \times 10^{-12} \mu_{B}$ [75]. In the case of Majorana neutrinos, the resonant neutrino-antineutrino conversions called "resonant spin-flavor" (RSF) conversions are studied in CCSNe [77-87]. Such resonant flavor conversions are induced by the finite neutrino magnetic moment in strong magnetic fields, and significant $\nu-\bar{\nu}$ transitions occur at the resonance baryon densities (see, e.g., Ref. [83]). The RSF conversions are sensitive to the sign of $2 Y_{e}-1$ [87], where the $Y_{e}$ is the electron fraction of the supernova material.

The neutrino-antineutrino oscillations considering neutrino-neutrino interactions are studied in Refs. [88-92]. The flavor equilibration of neutrino-antineutrino oscillations occurs in the scale determined by a neutrino magnetic potential in strong magnetic fields [91]. Such an equilibration phenomenon is different from the RSF conversion and suppressed by strong neutrino-neutrino interactions. However, the role of matter potentials on the neutrinoantineutrino oscillations is still unknown. The matter suppression, as confirmed in multiangle calculations, should be important in dense background matter.

In this work, we study the effect of matter suppression on equilibrations of neutrino-antineutrino oscillations caused by magnetic fields and discuss the possibility of such curious oscillations in astrophysical sites. In Sec. II, we carry out numerical simulations of the neutrino-antineutrino oscillations by assuming strong magnetic field $\left(B \gtrsim 10^{14} \mathrm{G}\right)$ and typical values of neutrino potentials in CCSNe and neutron-star mergers. From the simulations, we reveal the mechanism of matter suppression on the neutrinoantineutrino oscillations and propose a necessary condition of a magnetic potential to realize the equilibration of neutrino-antineutrino oscillations in dense matter. In Sec. III, we verify that the necessary condition of the neutrino-antineutrino oscillations given in Sec. II is satisfied outside a PNS in CCSNe based on supernova hydrodynamic simulations. Finally, our results are summarized in Sec. IV.

\section{SIMULATIONS OF FLAVOR EQUILIBRATION IN MAGNETIC FIELDS}

In the case of Majorana neutrinos, we perform simulations of the neutrino-antineutrino oscillations in magnetic fields by changing values of the MSW matter potential and the electron fraction based on a numerical setup of Ref. [91]. Equilibrium values of diagonal components of neutrino density matrices are reproduced analytically in the Appendix A.

\section{A. Equations of motion of flavor conversions}

Here, we employ the "neutrino line model" $[93,94]$ to simulate neutrino-antineutrino oscillations in magnetic fields. Flavor conversions of neutrinos with an emission angle $\theta$ at a radius $r$ are calculated by solving the Liouvillevon Neumann equation [91,93,94],

$$
\cos \theta \frac{\partial}{\partial r} D=-i[H, D]
$$

where $D(r, \theta)$ and $H(r, \theta)$ are $6 \times 6$ neutrino density matrix and Hamiltonian, respectively. The density matrix is given by

$$
D=\left(\begin{array}{cc}
\rho_{\theta} & X_{\theta} \\
X_{\theta}^{\dagger} & \bar{\rho}_{\theta}
\end{array}\right)
$$

where diagonal components $\rho_{\theta}$ and $\bar{\rho}_{\theta}$ are $3 \times 3$ density matrices of neutrinos and antineutrinos, respectively [91]. The subscript, $\theta$, means $\theta$ dependence. Here, we impose normalization of neutrino density matrices, $\operatorname{tr}\left[\rho_{\theta}+\bar{\rho}_{\theta}\right]=1$, where the "tr" represents the trace of a $3 \times 3$ matrix [e.g., $\left.\operatorname{tr}\left[\rho_{\theta}\right]=\sum_{\alpha=e, \mu, \tau}\left(\rho_{\theta}\right)_{\alpha \alpha}\right]$. The nondiagonal component $X_{\theta}$ is a correlation between $\nu$ and $\bar{\nu}$, which is usually negligible without a magnetic field. A finite value of $X_{\theta}$ induces neutrino-antineutrino oscillations. The Hamiltonian of neutrinos are decomposed into three terms:

$$
H(r, \theta)=H_{\mathrm{vac}}+H_{\text {matter }}+H_{\nu \nu} .
$$

The first term on the right-hand side represents the vacuum Hamiltonian in the magnetic field $[82,83,87,89,90]$,

$$
\begin{gathered}
H_{\mathrm{vac}}=\left(\begin{array}{cc}
\Omega(E) & V_{\mathrm{mag}} \\
-V_{\mathrm{mag}} & \Omega^{*}(E)
\end{array}\right), \\
V_{\mathrm{mag}}=B_{T}\left(\begin{array}{ccc}
0 & \mu_{e \mu} & \mu_{e \tau} \\
-\mu_{e \mu} & 0 & \mu_{\mu \tau} \\
-\mu_{e \tau} & -\mu_{\mu \tau} & 0
\end{array}\right),
\end{gathered}
$$

where $\mu_{\alpha \beta}(\alpha, \beta=e, \mu, \tau)$ are neutrino magnetic moments and $B_{T}$ is a transverse component of the magnetic field perpendicular to the direction of the neutrino emission. We assume the same neutrino magnetic moment irrespective of flavor dependence: $\mu_{e \mu}=\mu_{e \tau}=\mu_{\mu \tau}=\mu_{\nu}$. The diagonal component $\Omega(E)$ in Eq. (4) is a $3 \times 3$ vacuum Hamiltonian of neutrinos without a magnetic field [45]. Here, we use the same neutrino mixing parameter set, 
$\left\{\Delta m_{21}^{2}, \Delta m_{32}^{2}, \theta_{12}, \theta_{13}, \theta_{23}, \delta_{C P}\right\}$, as that of Ref. [45]. We set normal neutrino mass hierarchy $\left(\Delta m_{32}^{2}>0\right)$ in all of our simulations. We investigate oscillation behaviors of single energy neutrinos: $E=1 \mathrm{MeV}$. The flavor equilibration phenomenon in the scale of approximately $\left(\mu_{\nu} B_{T}\right)^{-1}$ does not depend on the choice of neutrino mixing parameters and neutrino energy qualitatively. The second term on the right-hand side of Eq. (3) is the matter potential $[82,83,87,89,90]$. It is given by

$$
\begin{gathered}
H_{\text {matter }}=\left(\begin{array}{cc}
V_{\text {matter }} & 0 \\
0 & -V_{\text {matter }}
\end{array}\right), \\
V_{\text {matter }}=-\frac{\lambda_{n}}{2} I_{3 \times 3}+\lambda_{e}\left(\begin{array}{ccc}
1 & 0 & 0 \\
0 & 0 & 0 \\
0 & 0 & 0
\end{array}\right), \\
\lambda_{e(n)}=\sqrt{2} G_{F} n_{e(n)},
\end{gathered}
$$

where $n_{e(n)}$ is the number density of electrons (neutrons) and $I_{3 \times 3}=\operatorname{diag}(1,1,1)$. The first term on the right-hand side of Eq. (7) does not contribute to flavor conversions without magnetic field. On the other hand, such a term plays an important role in neutrino-antineutrino oscillations caused by magnetic field effect.

The third term on the right-hand side of Eq. (3) shows the potential of neutrino-neutrino interactions which are sources of CNO. Here, we consider single energy neutrinos and focus on angular dependence of neutrinos by using the neutrino line model $[93,94]$. The potential of neutrinoneutrino interactions in this model is written as

$$
\begin{gathered}
H_{\nu \nu}=\left(\begin{array}{cc}
V_{\nu \nu} & 0 \\
0 & -V_{\nu \nu}^{*}
\end{array}\right), \\
V_{\nu \nu}=\sqrt{2} G_{F} n_{\nu} \int_{-\theta_{\max }}^{\theta_{\max }} \mathrm{d} \theta^{\prime}\left(1-\cos \left(\theta-\theta^{\prime}\right)\right) \\
\times\left\{\left(\operatorname{tr}\left[\rho_{\theta^{\prime}}\right]-\operatorname{tr}\left[\bar{\rho}_{\theta^{\prime}}^{*}\right]\right) I_{3 \times 3}+\left(\rho_{\theta^{\prime}}-\bar{\rho}_{\theta^{\prime}}^{*}\right)\right\}, \\
n_{\nu}=\sum_{\alpha=e, \mu, \tau}\left(n_{\nu_{\alpha}}+n_{\bar{\nu}_{\alpha}}\right),
\end{gathered}
$$

where $\theta_{\max }$ is the maximum neutrino emission angle in the line model [91]. The $n_{\nu}$ is a summation of initial number densities of all species of neutrinos. The initial values of diagonal terms of neutrino density matrices are given by $\left(\rho_{\theta}\right)_{\alpha \alpha}=n_{\nu_{\alpha}} / n_{\nu},\left(\bar{\rho}_{\theta}\right)_{\alpha \alpha}=n_{\bar{\nu}_{\alpha}} / n_{\nu}(\alpha=e, \mu, \tau)$.

\section{B. Flavor equilibrations in different $\left(\lambda_{e}, Y_{e}\right)$}

We perform simulations of neutrino-antineutrino oscillations based on numerical setup in Sec. II A. The initial neutrino number density is given by $n_{\bar{\nu}_{e}} / n_{\nu_{e}}=0.7$ and $n_{\nu_{x}} / n_{\nu_{e}}=n_{\bar{\nu}_{x}} / n_{\nu_{e}}=0.4(x=\mu, \tau)$ as done in Ref. [91]. In addition, we fix the strength of the nonlinear potential and that of magnetic potential as $\sqrt{2} G_{F} n_{\nu_{e}}=10^{-2} \mathrm{~cm}^{-1}$ and $\Omega_{\mathrm{mag}}=\mu_{\nu} B_{T}=0.1 \mathrm{~cm}^{-1}$. The strength of the matter potential $\lambda_{e}$ and the value of electron fraction $Y_{e}$ are variables for our simulations. The corresponding baryon density to a given $\lambda_{e}$ is $\rho_{b}=5 \times 10^{7} \mathrm{~g} / \mathrm{cm}^{3}\left(Y_{e} / 0.5\right)^{-1}\left(\lambda_{e} / 0.1 \mathrm{~cm}\right)$. Nondiagonal components in Eq. (2) are set to zero at $r=0$. In our simulations, we set a maximum emission angle to $\theta_{\max }=\pi / 3$.

The top (bottom) panel of Fig. 1 shows the evolution of angle averaged ratios of $\nu_{e}\left(\bar{\nu}_{e}\right)$ in a fixed value of the electron fraction $Y_{e}=0.45$ and different values of $\lambda_{e}$. Such angular average diagonal components are defined by

$$
\begin{aligned}
& \left\langle\rho_{e e}\right\rangle=\frac{3}{2 \pi} \int_{-\frac{\pi}{3}}^{\frac{\pi}{3}} \mathrm{~d} \theta \rho_{e e, \theta}, \\
& \left\langle\bar{\rho}_{e e}\right\rangle=\frac{3}{2 \pi} \int_{-\frac{\pi}{3}}^{\frac{\pi}{3}} \mathrm{~d} \theta \bar{\rho}_{e e, \theta} .
\end{aligned}
$$
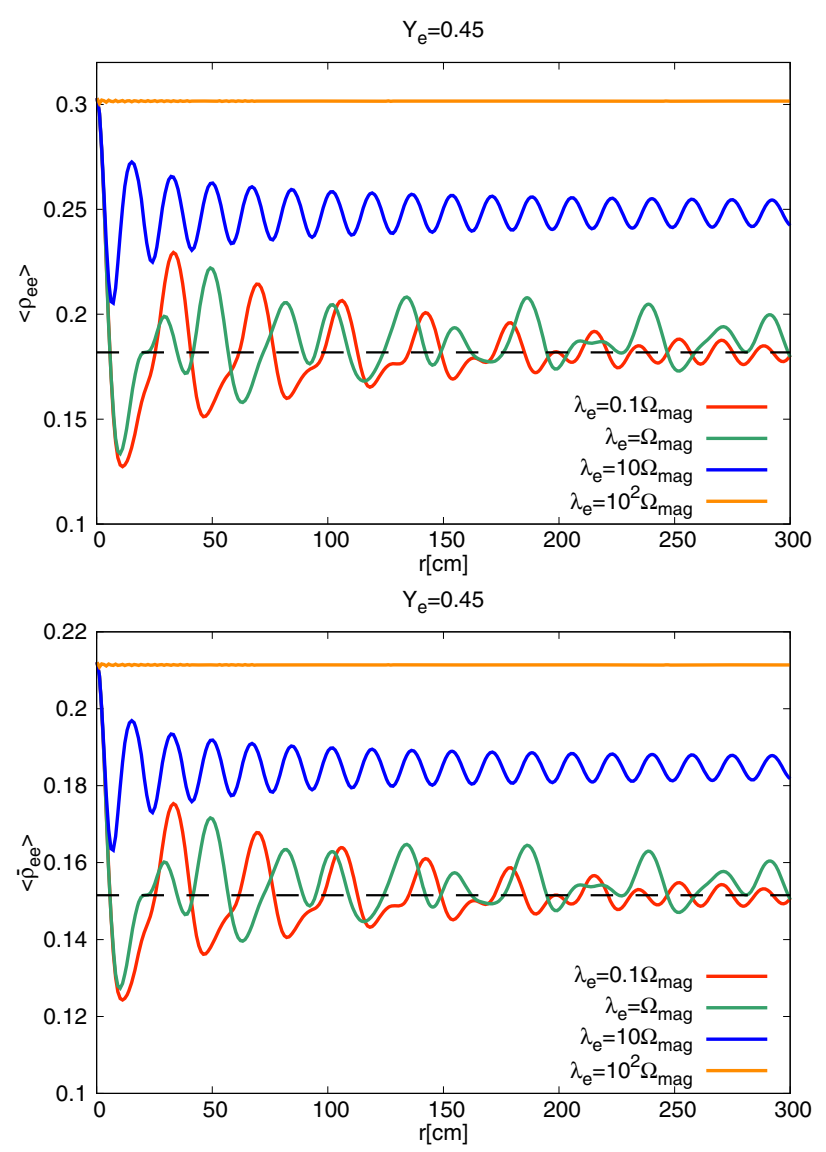

FIG. 1. The top panel shows evolution of $\left\langle\rho_{e e}\right\rangle$ as defined in Eq. (12) in different values of matter potential $\lambda_{e}$ with a constant value of electron fraction $Y_{e}=0.45$. The value of a black dashed line in the top panel corresponds to $\left(n_{\nu_{e}}+2 n_{\nu_{x}}\right) / 3 n_{\nu}=2 / 11$, which represents an equilibrium value of $\left\langle\rho_{e e}\right\rangle$. The bottom panel shows the case of $\left\langle\bar{\rho}_{e e}\right\rangle$ in Eq. (13). The value of the black dashed line in the bottom panel is $\left(n_{\bar{\nu}_{e}}+2 n_{\nu_{x}}\right) / 3 n_{\nu}=5 / 33$. 
The red and green curves in Fig. 1 represent the equilibration of neutrino-antineutrino oscillations when the potential of the magnetic field is no smaller than the matter potential: $\Omega_{\mathrm{mag}} \geq \lambda_{e}$. Such results are consistent with the equilibrium of three flavor neutrinos shown in Ref. [91]. The wavelength of the neutrino-antineutrino oscillations is the order of approximately $\Omega_{\mathrm{mag}}^{-1}=10 \mathrm{~cm}$. In such a strong magnetic field, all flavors of neutrinos and antineutrinos couple with flavor conversions. The black dashed lines in the top and the bottom panels of Fig. 1 represent equilibrium values of three flavor conversions. Such equilibrium values of three flavor conversions are reproduced in Appendix A 3, analytically. In the strong magnetic field, $\Omega_{\mathrm{mag}}$ becomes dominant in the neutrino Hamiltonian. The large $\Omega_{\text {mag }}$ plays an important role in increasing an amplitude of a correlation matrix $X_{\theta}$ in Eq. (2). Especially, a finite value of $X_{x y}\left(X_{y x}\right)$ which shows a correlation between $\nu_{x}\left(\nu_{y}\right)$ and $\bar{\nu}_{y}\left(\bar{\nu}_{x}\right)$ enables active three flavor neutrino-antieneutrino oscillations. Here, flavors $x$ and $y$ are given by rotation of flavor basis [34]. The detail mechanism of such three flavor oscillations in the magnetic field is shown in Appendix A 3.

In the case of $\lambda_{e}=10 \Omega_{\mathrm{mag}}$ (blue lines in Fig. 1), on the other hand, the neutrino-antineutrino oscillations occur, but such flavor conversions are suppressed because of the large matter potential. The flavor conversions do not reach the equilibrium values (black dash lines in Fig. 1). In the case of more dense matter $\lambda_{e}=10^{2} \Omega_{\mathrm{mag}}$ (dark orange lines in Fig. 1), values of $\left\langle\bar{\rho}_{e e}\right\rangle$ and $\left\langle\rho_{e e}\right\rangle$ are constant irrespective of the radius, so the flavor conversions are completely suppressed. Such results indicate that we need to take into account the contributions of matter potential in order to study the neutrino-antineutrino oscillations in the strong magnetic fields of explosive astrophysical sites. The mechanism of matter suppression as shown in Fig. 1 is discussed in Appendix A 1. Here, we set a constant value of $Y_{e}=0.45$, so the matter potential $\left|\lambda_{e}-\lambda_{n}\right| \propto\left|2 Y_{e}-1\right|$ becomes finite and contributes to the matter suppression. However, the matter potential is sensitive to the value of $Y_{e}$, so matter suppression should also depend on the value of $Y_{e}$.

Figure 2 shows the dependence of electron fraction $Y_{e}$ in the neutrino-antineutrino oscillations. The MSW matter potential is fixed by $\lambda_{e}=10^{2} \Omega_{\mathrm{mag}}$. Any flavor conversions are suppressed in the cases of $Y_{e}=0.45$ (red solid lines) and $Y_{e}=0.55$ (magenta solid lines). On the other hand, flavor conversions become prominent as the value of $Y_{e}$ is close to 0.5 (see green and dark-orange solid lines). The matter suppression disappears in the case of $Y_{e}=0.5$ (blue solid lines). Here, we fix the strength of matter potential as $\lambda_{e}=10^{2} \Omega_{\mathrm{mag}}$, but flavor conversions at $Y_{e}=0.5$ also appear, even though more dense matter potential such as $\lambda_{e}=10^{3} \Omega_{\mathrm{mag}}$ is employed. The matter suppression disappears at $Y_{e}=0.5$ because matter potentials of charged
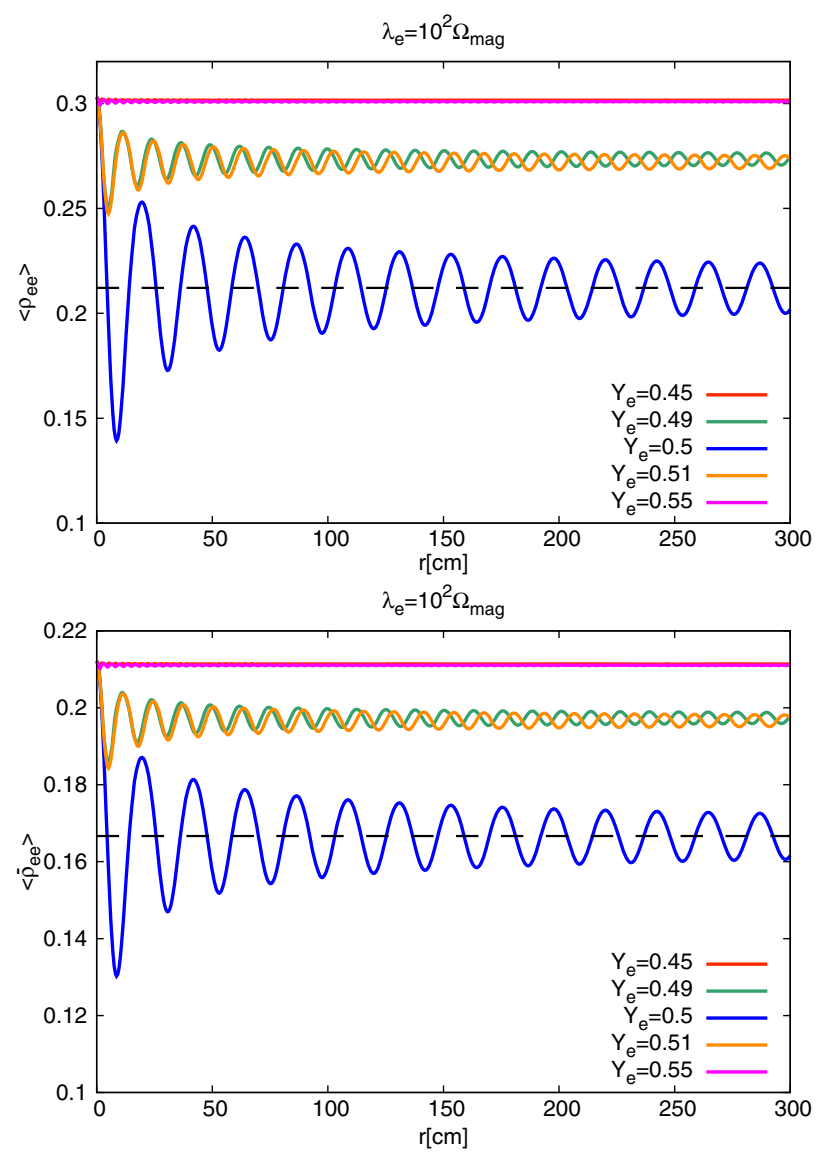

FIG. 2. The neutrino-antineutrino oscillations as shown in Fig. 1 in different values of electron fraction $Y_{e}$ around 0.5. The strength of MSW matter potential is fixed by $\lambda_{e}=10^{2} \Omega_{\mathrm{mag}}$. The black dashed line in the top panel corresponds to $\left(n_{\nu_{e}}+n_{\nu_{x}}\right) / 2 n_{\nu}=7 / 33$. The black dashed line in the bottom panel shows $\left(n_{\bar{\nu}_{e}}+n_{\nu_{x}}\right) / 2 n_{\nu}=1 / 6$.

and neutral current reactions are canceled out: $\lambda_{e}=\lambda_{n}$. The black dash line in the top (bottom) panel of Fig. 2 shows an equilibrium value of $\left\langle\rho_{e e}\right\rangle\left(\left\langle\bar{\rho}_{e e}\right\rangle\right)$ at $Y_{e}=0.5$. These equilibrium values are different from that in Fig. 1 because one of flavors is decoupled from flavor conversions. The matter term in Eq. (7) induces two types of matter potentials such as $\left|\lambda_{e}-\lambda_{n}\right|$ and $\lambda_{n}$ in the equation of motion of neutrino density matrices. The $\left|\lambda_{e}-\lambda_{n}\right|$ disappears at $Y_{e}=0.5$, but another matter potential $\lambda_{n}$ is not eliminated. The large $\lambda_{n}$ results in decoupling of $\nu_{x}$ and $\bar{\nu}_{x}$ in flavor conversions. The detail of such one flavor decoupling is shown in Appendix A 2, and equilibrium values in Fig. 2 are reproduced analytically. Our simulations in a parameter space of $\left(\lambda_{e}, Y_{e}\right)$ indicate that the strong magnetic field potential $\Omega_{\mathrm{mag}}$,

$$
\Omega_{\mathrm{mag}} \geq \lambda
$$

is necessary for the equilibration of neutrino-antineutrino oscillations where we define $\lambda=\left|\lambda_{e}-\lambda_{n}\right|$. The above 
condition can be satisfied even in dense astrophysical sites when $Y_{e}$ in the dense matter is close to 0.5 .

\section{POSSIBILITY OF SUPERNOVA NEUTRINO-ANTINEUTRINO OSCILLATIONS IN MAGNETIC FIELDS}

The necessary condition of the neutrino-antineutrino oscillations proposed in our simulations is extended by taking account of the contribution from neutrino-neutrino interactions inside CCSNe. We investigate the possibility of the flavor equilibrium of neutrino-antineutrino oscillations in CCSNe by employing matter profiles and neutrino spectra obtained in a hydrodynamic simulation of CCSNe of a $11.2 M_{\odot}$ progenitor. The case of electron capture supernova (ECSN) is shown in Appendix B.

\section{A. Strength of potentials in neutrino Hamiltonian}

As suggested in Eq. (14), the occurrence of the neutrinoantineutrino oscillations can be evaluated by a comparison of $\Omega_{\mathrm{mag}}=\mu_{\nu} B_{T}$ with other potentials in neutrino Hamiltonian. We focus on the neutrino-antineutrino oscillations near the PNS $\left[r \sim \mathcal{O}\left(10-10^{3}\right) \mathrm{km}\right]$ where the magnetic field may be large enough to satisfy Eq. (14). To discuss the possibility of such flavor conversions in magnetic fields, we need matter profiles and neutrino spectra of deep inside CCSNe. We employ different time snapshots of matter profiles and neutrino spectra obtained in a hydrodynamic simulation of supernova explosions. The neutrino radiation-hydrodynamic simulations are performed by 3DnSNe-IDSA code [95] (see Ref. [96] for hydrodynamic method, Refs. [97,98] for neutrino transport, and Ref. [99] for code comparison). The initial condition is taken from $11.2 M_{\odot}$ progenitor model of Woosley et al. [100]. The hydrodynamic profiles of this model are also used in Ref. [101], the detailed setup of which is written in Ref. [48].

Since we employ the profile that is taken from the pure hydrodynamic calculation, there is no spacial profile of the magnetic field in our explosion model. We assume a transverse component of a dipole magnetic field whose radial dependence is given by $B_{T} \propto r^{-3}$ as employed in previous works $[82,83,87]$,

$$
B_{T}=B_{0}\left(\frac{R_{\nu}}{r}\right)^{3}
$$

where $R_{\nu}$ is a radius of the neutrino sphere and $B_{0}$ is a strength of a transverse magnetic field to the direction of neutrino emission at $r=R_{\nu}$. Here, we set $R_{\nu}=30 \mathrm{~km}$ and focus on neutrino emission along the radial direction from the equator of the PNS. We fix the value of the neutrino magnetic moment, $\mu_{\nu}=10^{-12} \mu_{B}$, satisfying the current upper limit of neutrino magnetic moments. The potential of the magnetic field interaction is written as $\Omega_{\mathrm{mag}}=\mu_{\nu} B_{T}$, so the neutrino-antineutrino oscillations would be induced even in the case of smaller value of $\mu_{\nu}$ than $10^{-12} \mu_{B}$ if we set a larger magnetic field, $B_{0}$, on the surface of neutrino sphere.

Neutrino-neutrino interactions should be taken into account in neutrino oscillations near the PNS. Behaviors of the neutrino-antineutrino oscillations in a magnetic field are sensitive to the strength of neutrino-neutrino interactions. The large potential of neutrino-neutrino interactions also prevents flavor conversions if the potential of the magnetic field is much smaller than that of neutrinoneutrino interactions [91]. If the neutrino emission angle is large, the strength of neutrino-neutrino interactions can be characterized by $\sqrt{2} G_{F}\left|n_{\nu_{e}}-n_{\bar{\nu}_{e}}\right|$ without considering a contribution from an angle factor $1-\cos \left(\theta-\theta^{\prime}\right)$ in Eq. (10). However, such an angle factor cannot be ignored when the maximum neutrino emission angle is small. The strength of neutrino-neutrino interactions depends on the value of the maximum neutrino emission angle. We use the bulb model [31] to determine the strength of neutrinoneutrino interactions outside a neutrino sphere. In the bulb model, the maximum neutrino emission angle at the radius $r$ is given by $\sin \theta_{\max }=R_{\nu} / r$. The potential of neutrinoneutrino interactions in Eq. (10) is a function of neutrino emission angle $\theta$. Such angle dependence is removed by assuming $\theta=0$ under the single angle approximation [31]. The strength of neutrino-neutrino interactions including the angular factor is described by

$$
\begin{aligned}
\zeta & =\frac{\sqrt{2} G_{F}}{2 \pi R_{\nu}^{2}}\left|\frac{L_{\nu_{e}}}{\left\langle E_{\nu_{e}}\right\rangle}-\frac{L_{\bar{\nu}_{e}}}{\left\langle E_{\bar{\nu}_{e}}\right\rangle}\right| \int_{\cos \theta_{\max }}^{1} \mathrm{~d} \cos \theta(1-\cos \theta) \\
& =\frac{\sqrt{2} G_{F}}{4 \pi R_{\nu}^{2}}\left|\frac{L_{\nu_{e}}}{\left\langle E_{\nu_{e}}\right\rangle}-\frac{L_{\bar{\nu}_{e}}}{\left\langle E_{\bar{\nu}_{e}}\right\rangle}\right|\left(1-\sqrt{1-\left(\frac{R_{\nu}}{r}\right)^{2}}\right)^{2}
\end{aligned}
$$

where $L_{\nu_{i}}$ and $\left\langle E_{\nu_{i}}\right\rangle$ are the luminosity and mean energy of $\nu_{i}\left(\nu_{i}=\nu_{e}, \bar{\nu}_{e}\right)$ on the surface of the neutrino sphere. This strength is almost proportional to $r^{-4}$ for large radius $\left(r \gg R_{\nu}\right)$. The small asymmetry between $\nu_{e}$ number flux and that of $\bar{\nu}_{e}$ on the surface of the neutrino sphere would be favorable for the neutrino-antineutrino oscillations because of the small value of $\zeta$.

In outer layers of the supernova material $\left[r \geq \mathcal{O}\left(10^{3}\right)\right.$ $\mathrm{km}]$, neutrino-neutrino interactions become small, and the matter potential is comparable to the vacuum Hamiltonian, which induces the RSF conversions [82,83,87]. The contribution from the vacuum potential is no longer negligible in such an outer region. Therefore, the neutrinoantineutrino oscillations may be negligible if the strength of vacuum Hamiltonian is larger than that of magnetic field potential. The necessary condition for the equilibration of neutrino-antineutrino oscillations in CCSNe can be summarized as 


$$
\begin{aligned}
\Omega_{\mathrm{mag}} & \geq \eta, \\
\eta & =\max \{\lambda, \zeta, \omega\},
\end{aligned}
$$

where $\omega=\left|\Delta m_{32}^{2}\right| / 2 E$ is the atmospheric vacuum frequency which characterizes the strength of vacuum Hamiltonian. We impose a typical mean energy of supernova neutrinos, $E=10 \mathrm{MeV}$, on $\omega$.

\section{B. Comparison of potentials in a CCSN model}

The strengths of neutrino potentials in different explosion phases are shown in Fig. 3. The top panel shows the case of the early explosion phase at $31 \mathrm{~ms}$ postbounce. The strength of the matter potential $\lambda$ is the largest near the surface of the PNS, so $\eta=\lambda$ is satisfied, and the blue solid line completely corresponds to the red dotted line in the top panel of Fig. 3. The decrease of $\lambda$ around $100 \mathrm{~km}$ corresponds to the reduction of the baryon density outside the shock wave. There is a boundary between the iron core and Si layer around $400 \mathrm{~km}$. The matter potential $\lambda \propto$ $\left|2 Y_{e}-1\right|$ decreases rapidly outside the Si layers because of
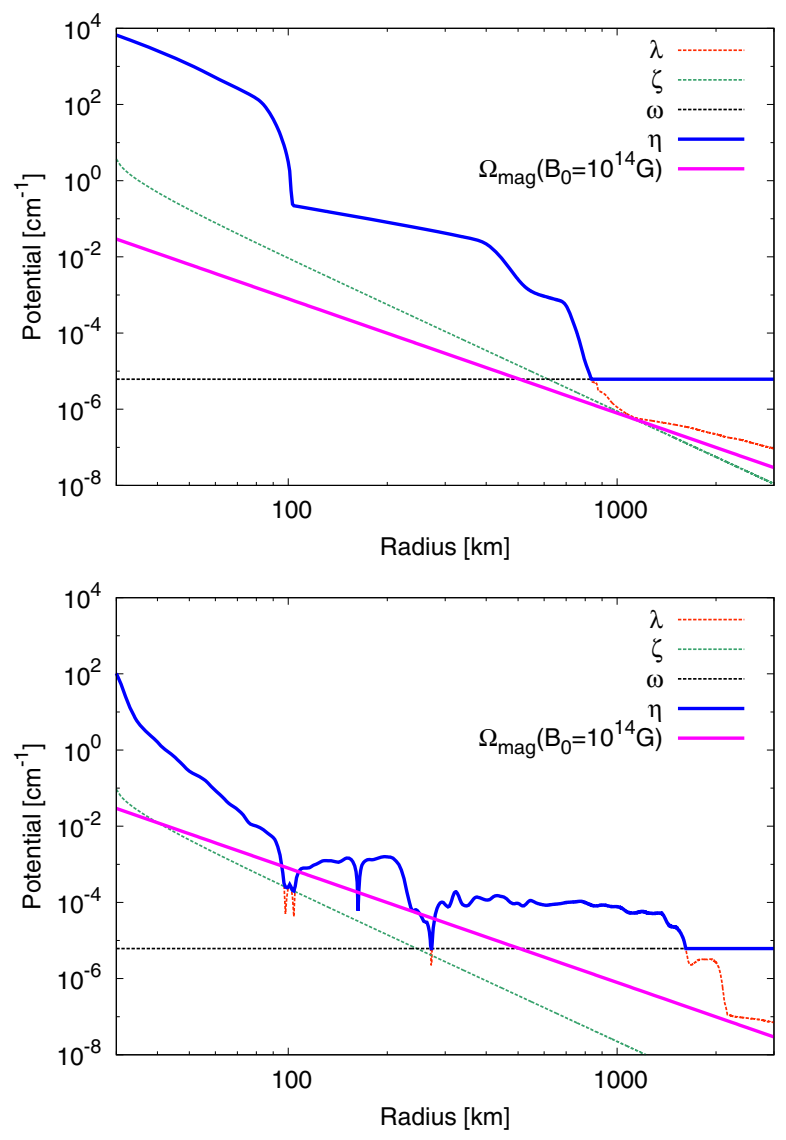

FIG. 3. The top (bottom) panel shows strengths of neutrino potentials at 31 (331) ms postbounce. The potential of the magnetic field (magenta solid line) is given by assuming $B_{0}=$ $10^{14} \mathrm{G}$ and $\mu_{\nu}=10^{-12} \mu_{B}$. The necessary condition of neutrinoantineutrino oscillations in Eq. (17) is realized when $\eta$ (blue solid line) becomes smaller than $\Omega_{\mathrm{mag}}$ (magenta solid line).
$Y_{e} \sim 0.5$. The matter potential becomes smaller in the outer region and finally comparable with the vacuum potential. In outer region $(r>840 \mathrm{~km})$, the vacuum potential becomes dominant in the neutrino Hamiltonian, and $\eta$ corresponds to $\omega$. A magenta solid line in the top panel of Fig. 3 represents a radial profile of $\Omega_{\mathrm{mag}}=\mu_{\nu} B_{T}$ assuming $B_{0}=10^{14} \mathrm{G}$ in Eq. (15). The necessary condition of the neutrino-antineutrino oscillations is satisfied where the value of $\Omega_{\mathrm{mag}}$ (magenta solid line) is larger than that of $\eta$ (blue solid line). In the top panel of Fig. 3, there is no crossing of these two solid lines, so Eq. (17) is not realized at $31 \mathrm{~ms}$ postbounce in the case of $B_{0}=10^{14} \mathrm{G}$. The dense matter profile in the early explosion phase raises up the value of $\eta$ near the PNS. The potential $\zeta$ is not a dominant term in the early explosion phase of standard CCSNe having iron cores in the progenitors, but we can not ignore the contribution from neutrino-neutrino interactions in the case of an ECSN as shown in Appendix B. The line of $\Omega_{\mathrm{mag}}$ is shifted upward by increasing the value of $B_{0}$. The top panel of Fig. 3 indicates that a stronger magnetic field on the surface of neutrino sphere $\left(B_{0}>10^{14} \mathrm{G}\right)$ is required for the neutrino-antineutrino oscillations in the early explosion phase.

The radial profiles of potentials at $331 \mathrm{~ms}$ postbounce are shown in the bottom panel of Fig. 3. The values of $\lambda$ and $\zeta$ in the bottom panel of Fig. 3 are smaller than those in the top panel of Fig. 3 because the baryon density and neutrino fluxes near the PNS decrease as the explosion time has passed. The small values of these potentials enable the crossing of $\eta$ (blue solid line) and $\Omega_{\text {mag }}$ (magenta solid line) in the bottom panel of Fig. 3. The strong magnetic field on the surface of neutrino sphere $\left(B_{0}>10^{14} \mathrm{G}\right)$ is enough to fulfill Eq. (17) at $331 \mathrm{~ms}$ postbounce. There are several peaks in $\lambda$ (red dotted line) which correspond to regions of $Y_{e} \sim 0.5$. The value of $Y_{e}$ in the supernova material is changed by a heating of the shock wave propagation. Neutrino-neutrino interactions become prominent in the region of $Y_{e} \sim 0.5$, which prevents the reduction of $\eta$.

To satisfy Eq. (17) at a radius $r$, the magnetic field on the surface of neutrino sphere $B_{0}$ should be larger than $B_{0, \min }(r)$ written as

$$
B_{0, \min }(r)=\frac{r^{3}}{\mu_{\nu} R_{\nu}^{3}} \eta
$$

The radial profiles of $B_{0, \min }(r)$ at the early and the later explosion phases are shown in Fig. 4. As shown in the case of $33 \mathrm{~ms}$ (red solid line), the minimum value of $B_{0, \min }(r)$ is given by $4.52 \times 10^{14} \mathrm{G}$ at $r=836 \mathrm{~km}$. Therefore, $B_{0}$ should be larger than $4.52 \times 10^{14} \mathrm{G}$ to fulfill Eq. (17) in the early explosion phase. On the other hand, in the case of $331 \mathrm{~ms}$ (green solid line), small baryon density near the PNS reduces the value of $\eta$, which results in smaller value of $B_{0, \min }(r)$ than that in the early phase. Several peaks of $\lambda$ as shown in the bottom panel of Fig. 3 induce small values 


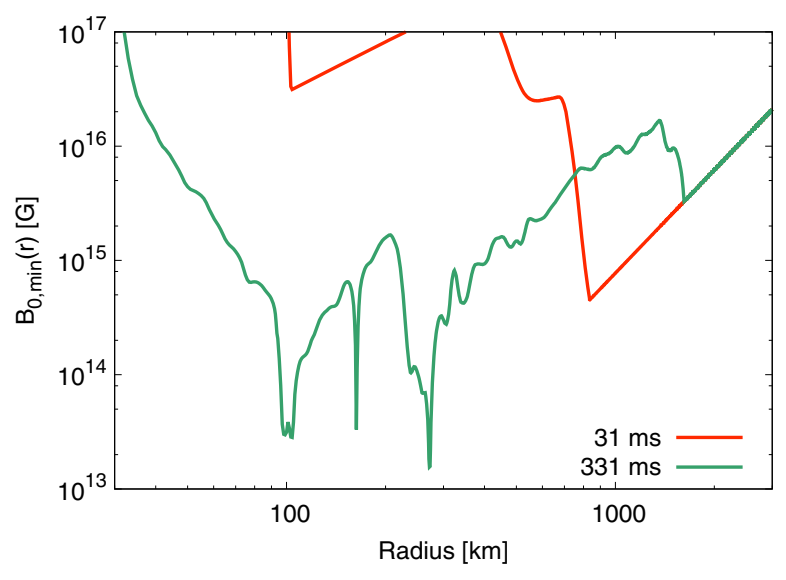

FIG. 4. Radial profiles of $B_{0, \min }(r)$ at $31 \mathrm{~ms}$ and $331 \mathrm{~ms}$ postbounce. $B_{0, \min }(r)$ is defined in Eq. (18). The magnetic field at $r=R_{\nu}\left(B_{0}\right)$ should be larger than $4.52 \times 10^{14}\left(1.56 \times 10^{13}\right) \mathrm{G}$ in order to satisfy Eq. (17) at 31(331) ms.

of $B_{0, \min }(r)$ in Fig. 4. The necessary condition of Eq. (17) is satisfied in the later explosion phase if $B_{0}$ is larger than $1.56 \times 10^{13} \mathrm{G}$. The required magnetic field for the neutrino-antineutrino oscillations becomes smaller in the later explosion phase, so the later explosion phase would be favorable to observe the neutrino-antineutrino oscillations. Such a feature would be general and independent of progenitor models. The case of ECSN is shown in Appendix B.

\section{CONCLUSIONS}

We study neutrino-antineutrino oscillations caused by a strong magnetic field in dense matter in the case of Majorana neutrinos. Numerical simulations of such neutrino-antineutrino oscillations are carried out by changing values of baryon densities and electron fractions. We reveal that the neutrino-antineutrino oscillations are sensitive to strengths of matter potentials such as $\left|\lambda_{e}-\lambda_{n}\right|$ and $\lambda_{n}$. The flavor equilibration occurs when the strength of magnetic field potential is comparable with that of a matter potential: $\Omega_{\mathrm{mag}} \sim \lambda_{n}$. Equilibrium states of such three flavor neutrinoantineutrino oscillations are consistent with numerical results in Ref. [91]. On the other hand, in the case of more dense matter $\left(\left|\lambda_{e}-\lambda_{n}\right|, \lambda_{n} \gg \Omega_{\mathrm{mag}}\right)$, any flavor conversions are suppressed because correlations between neutrinos and antineutrinos fail to grow up in large matter potentials. The values of matter potentials depend on both the baryon density and electron fractions inside material. We find that the flavor equilibration also appears around $Y_{e} \sim 0.5$, irrespective of large baryon densities. One of the neutrino flavors is decoupled from flavor conversions around $Y_{e} \sim 0.5$ in dense matter. The values of equilibrium states of $\left\langle\rho_{e e}\right\rangle$ and $\left\langle\bar{\rho}_{e e}\right\rangle$ are different from those in three flavor conversions in neutron- (proton-)rich matter.

The mechanism of the neutrino-antineutrino oscillations in strong magnetic fields, which we focus on here, is different from that of RSF. In our simulations, the matter potential is much higher than the vacuum Hamiltonian, so that there is no resonance like RSF. The equilibration of neutrino-antineutrino oscillations in our simulations is independent of neutrino mass hierarchy and neutrino energy. Such neutrino-antineutrino oscillations are sensitive to the strength of neutrino-neutrino interactions [91]. As shown in Appendix A, however, the flavor equilibration of neutrino-antineutrino oscillations is possible even in the absence of neutrino-neutrino interactions. The origin of the neutrino-antineutrino oscillations is the coupling of matter potentials with the magnetic field potential.

We verify that a necessary condition for the equilibration of neutrtino-antineutrino oscillations is satisfied in a 11.2 $M_{\odot}$ CCSN model. To satisfy such a condition, the magnetic field on the surface of the PNS should be larger than $10^{13} \mathrm{G}$ in the case of $\mu_{\nu}=10^{-12} \mu_{B}$, which is the same order as a tight upper limit of neutrino magnetic moment [74]. The strength of matter potential becomes smaller near the PNS as the explosion phase has passed, so the required magnetic field for the neutrino-antineutrino oscillations becomes smaller in the later explosion phase. Such a trend is also confirmed in ECSN, irrespective of different matter profiles from an iron core of the standard CCSNe (see Appendix B).

Our result indicates that the typical magnetic field of pulsars (approximately $10^{12} \mathrm{G}$ ) is not enough to induce the flavor equilibrium of neutrino-antineutrino oscillations near the PNS. On the other hand, the neutrino-antineutrino oscillations are possible in the typical magnetic field of magnetars (approximately $10^{14} \mathrm{G}$ ) [102]. The strength of the magnetic field potential $\Omega_{\mathrm{mag}}$ is proportional to neutrino magnetic moments and the transverse magnetic field, so a stronger magnetic field can induce the neutrinoantineutrino oscillations even in $\mu_{\nu}<10^{-12} \mu_{B}$. A supernova explosion scenario leaving a magnetar at the center potentially updates the current upper limit on $\mu_{\nu}$. Further quantitative studies discussing the magnetic field effect on neutrino detection will be required to identify possibilities to withdraw properties of neutrinos from supernova neutrinos. Here, we focus on specific supernova progenitor models, but the necessary condition of the neutrino-antineutrino oscillations proposed in this work can be applied in more general explosive phenomena such as neutron-star mergers and gamma-ray bursts.

\section{ACKNOWLEDGMENTS}

This study was supported in part by JSPS/MEXT KAKENHI Grants No. JP19J13632, No. JP18H01212, No. JP17H06364, and No. JP21H01088. This work is also supported by the National Institutes of Natural Sciences (NINS) program for cross-disciplinary study (Grants No. 01321802 and No. 01311904) on Turbulence, Transport, and Heating Dynamics in Laboratory and Solar/ 
Astrophysical Plasmas: "SoLaBo-X." Numerical computations were carried out on the PC cluster and Cray XC50 at the Center for Computational Astrophysics, National Astronomical Observatory of Japan. This research was also supported by MEXT as Program for Promoting research on the Supercomputer Fugaku (Toward a unified view of the universe: From large scale structures to planets) and Joint Institute for Computational Fundamental Science (JICFuS).

\section{APPENDIX A: MATTER SUPPRESSION IN NEUTRINO-ANTINEUTRINO OSCILLATIONS}

We discuss the mechanism of the flavor equilibration of neutrino-antineutrino oscillations in strong magnetic fields, which is confirmed in Figs. 1 and 2. Here, we assume that the neutrino-antineutrino oscillations are dominant and ordinary flavor conversions without magnetic fields are negligible. Furthermore, we consider flavor conversions in dense matter where a vacuum potential $\Omega(E)$ and a potential of neutrino-neutrino interactions are negligible. Such conditions are written as

$$
\begin{aligned}
{\left[H_{\nu}, \rho_{\theta}\right] } & \sim 0, \\
{\left[H_{\bar{\nu}}, \bar{\rho}_{\theta}\right] } & \sim 0, \\
H_{\nu} \sim H_{\bar{\nu}} & \sim-V_{\text {matter }},
\end{aligned}
$$

where $H_{\nu}\left(H_{\bar{\nu}}\right)$ is the Hamiltonian of neutrinos (antineutrinos) without the magnetic field potential. We focus on flavor conversions of neutrinos whose emission angle is $\theta=0$. The $\theta=0$ is imposed on Eq. (1), and the index $\theta$ in a neutrino density matrix is dropped hereafter. We investigate flavor conversions in the $e-x-y$ basis [34] instead of the flavor $e-\mu-\tau$ basis. The matter potential is invariant under the rotation from the flavor basis to the $e-x-y$ basis:

$$
\begin{gathered}
V_{\text {matter }}^{\prime}=R^{T}\left(\theta_{23}\right) V_{\text {matter }} R\left(\theta_{23}\right)=V_{\text {matter }} \\
R\left(\theta_{23}\right)=\left(\begin{array}{ccc}
1 & 0 & 0 \\
0 & \cos \theta_{23} & \sin \theta_{23} \\
0 & -\sin \theta_{23} & \cos \theta_{23}
\end{array}\right)
\end{gathered}
$$

On the other hand, the magnetic field potential does not commute with $R\left(\theta_{23}\right)$, so the matrix components are transformed by the rotation from the flavor basis to the $e-x-y$ basis,

$$
\begin{aligned}
V_{\mathrm{mag}}^{\prime} & =R^{T}\left(\theta_{23}\right) V_{\mathrm{mag}} R\left(\theta_{23}\right) \\
& =\Omega_{\mathrm{mag}}\left(\begin{array}{ccc}
0 & 0 & 1 \\
0 & 0 & 1 \\
-1 & -1 & 0
\end{array}\right),
\end{aligned}
$$

where $\Omega_{\mathrm{mag}}=\mu_{\nu} B_{T}$. The evolutions of neutrino density matrices in Eq. (1) are decomposed by

$$
\begin{gathered}
\partial_{r} \rho=-i\left[H_{\nu}, \rho\right]-i\left(V_{\mathrm{mag}} X^{\dagger}+X V_{\mathrm{mag}}\right), \\
\partial_{r} \bar{\rho}=-i\left[H_{\bar{\nu}}, \bar{\rho}\right]+i\left(V_{\mathrm{mag}} X+X^{\dagger} V_{\mathrm{mag}}\right), \\
\partial_{r} X=-i\left(H_{\nu} X-X H_{\bar{\nu}}+V_{\mathrm{mag}} \bar{\rho}-\rho V_{\mathrm{mag}}\right) .
\end{gathered}
$$

From Eqs. (A1)-(A7), evolutions of diagonal components of neutrino density matrices are described by

$$
\begin{gathered}
\partial_{r} \rho_{e e} \sim-2 \Omega_{\mathrm{mag}} X_{e y, i}, \\
\partial_{r} \rho_{x x} \sim-2 \Omega_{\mathrm{mag}} X_{x y, i}, \\
\partial_{r} \rho_{y y} \sim 2 \Omega_{\mathrm{mag}}\left(X_{y e, i}+X_{y x, i}\right), \\
\partial_{r} \bar{\rho}_{e e} \sim-2 \Omega_{\mathrm{mag}} X_{y e, i}, \\
\partial_{r} \bar{\rho}_{x x} \sim-2 \Omega_{\mathrm{mag}} X_{y x, i}, \\
\partial_{r} \bar{\rho}_{y y} \sim 2 \Omega_{\mathrm{mag}}\left(X_{e y, i}+X_{x y, i}\right),
\end{gathered}
$$

where $X_{\alpha \beta, i}(\alpha, \beta=e, x, y)$ represents the imaginary parts of $X_{\alpha \beta}$. From these equation of motion, we can find conservation laws,

$$
\begin{aligned}
& \rho_{e e}+\rho_{x x}+\bar{\rho}_{y y}=\text { const. } \\
& \bar{\rho}_{e e}+\bar{\rho}_{x x}+\rho_{y y}=\text { const. }
\end{aligned}
$$

which result in a decoupling of the $\nu_{e}-\nu_{x}-\bar{\nu}_{y}$ sector and the $\bar{\nu}_{e}-\bar{\nu}_{x}-\nu_{y}$ sector during the neutrino-antineutrino oscillations. The above conservation laws are actually confirmed in our numerical simulations. Here, we only focus on flavor conversions among $\nu_{e}, \nu_{x}$, and $\bar{\nu}_{y}$. Almost the same discussion is possible in the $\bar{\nu}_{e}-\bar{\nu}_{x}-\nu_{y}$ sector because of the decoupling in Eq. (A14). We require evolution of $X_{e y, i}$ and $X_{x y, i}$ in order to close Eqs. (A8), (A9), and (A13). Nondiagonal components such as $\rho_{e x}, \rho_{e y}$, $\bar{\rho}_{e x}$, and $\bar{\rho}_{e y}$ would be ignored under the assumptions of Eq. (A1). Therefore, equation of motion of $X_{e y}$ and $X_{x y}$ are given by

$$
\begin{gathered}
\partial_{r} X_{e y, r} \sim\left(\lambda_{e}-\lambda_{n}\right) X_{e y, i}, \\
\partial_{r} X_{e y, i} \sim-\left(\lambda_{e}-\lambda_{n}\right) X_{e y, r}-\Omega_{\mathrm{mag}}\left(\bar{\rho}_{y y}-\rho_{e e}\right), \\
\partial_{r} X_{x y, r} \sim-\lambda_{n} X_{x y, i}, \\
\partial_{r} X_{x y, i} \sim \lambda_{n} X_{x y, r}-\Omega_{\mathrm{mag}}\left(\bar{\rho}_{y y}-\rho_{x x}\right),
\end{gathered}
$$

where $X_{e y, r}\left(X_{x y, r}\right)$ are the real parts of $X_{e y}\left(X_{x y}\right)$, respectively. Numerical results of our simulations can be analyzed 
by comparing three different frequencies such as $\left|\lambda_{e}-\lambda_{n}\right|$, $\lambda_{n}$, and $\Omega_{\mathrm{mag}}$ in the above differential equations.

\section{Case of $\left|\lambda_{e}-\lambda_{n}\right|, \lambda_{n} \gg \Omega_{\mathrm{mag}}$}

In this case, the second term on the right-hand side of Eq. (A18) is almost negligible, so

$$
\partial_{r}^{2} X_{x y, i} \sim-\lambda_{n}^{2} X_{x y, i}
$$

is obtained. There is no correlation between neutrinos and antineutrinos at the beginning of the calculation, so $X_{x y, i}$ should be proportional to $\sin \left(\lambda_{n} r\right)$. The coefficient of $X_{x y, i}$ can be derived by substituting $X_{x y, i}$ for Eq. (A18) and considering the case of $r=0$. However, the right-hand side of Eq. (A18) is zero at $r=0$. Therefore, $X_{x y, i}$ is also zero, irrespective of the radius, which results in the decoupling of $\nu_{x}$ during the neutrino-antineutrino oscillations. It is also assumed that the $\left|\lambda_{e}-\lambda_{n}\right|$ is much larger than $\Omega_{\text {mag }}$. This means the value of $\left|2 Y_{e}-1\right|$ is finite. The derivation of $X_{e y, i}$ is carried out in analogy with $X_{x y, i}$. The value of $X_{e y, i}$ is almost zero because the second term on the right-hand side of Eq. (A16) is negligible. Therefore, matter suppression is dominant, and any flavor conversion does not appear. This analytical model can explain the strong matter suppression in our simulations [e.g., $\lambda_{e}=10^{2} \Omega_{\mathrm{mag}}$ (darkorange solid lines) in Fig. 1].

\section{Case of $\lambda_{e}=\lambda_{n}\left(Y_{e}=0.5\right), \lambda_{n} \gg \Omega_{\mathrm{mag}}$}

The $X_{x y, i}$ is negligible, and $\nu_{x}$ decouples from the flavor conversions as shown in Appendix A 1. The difference from the previous case is $\left|\lambda_{e}-\lambda_{n}\right|=0$ where the electron fraction $Y_{e}$ is equal to 0.5 . The finite $X_{e y, i}$ induces two flavor oscillations between $\nu_{e}$ and $\bar{\nu}_{y}$, irrespective of a large matter potential: $\lambda_{n} \gg \Omega_{\mathrm{mag}}$. The second derivative of $X_{e y, i}$ is described by

$$
\partial_{r}^{2} X_{e y, i} \sim-4 \Omega_{\mathrm{mag}}^{2} X_{e y, i},
$$

where $\left(\bar{\rho}_{y y}-\rho_{e e}\right)$ in Eq. (A16) is eliminated by multiplying the derivative $\partial_{r}$ and using Eqs. (A13) and (A8). The correlation $X_{e y, i}$ is derived by solving Eq. (A20) and imposing an initial condition of neutrino density matrices on Eq. (A16). In our simulations, the initial condition of the neutrino density matrix at $r=0$ is given by $\rho_{e e}=n_{\nu_{e}} / n_{\nu}$, $\rho_{x x}=n_{\nu_{x}} / n_{\nu}$, and $\bar{\rho}_{y y}=n_{\nu_{x}} / n_{\nu}$ where $n_{\nu}=n_{\nu_{e}}+n_{\bar{\nu}_{e}}+4 n_{\nu_{x}}$. Then, by solving Eqs. (A8) and (A13), we obtain

$$
\begin{aligned}
& \rho_{e e}=\frac{n_{\nu_{e}}}{n_{\nu}}+\frac{n_{\nu_{x}}-n_{\nu_{e}}}{2 n_{\nu}}\left(1-\cos 2 \Omega_{\mathrm{mag}} r\right), \\
& \rho_{x x}=\frac{n_{\nu_{x}}}{n_{\nu}}=\text { const. } \\
& \bar{\rho}_{y y}=\frac{n_{\nu_{x}}}{n_{\nu}}-\frac{n_{\nu_{x}}-n_{\nu_{e}}}{2 n_{\nu}}\left(1-\cos 2 \Omega_{\mathrm{mag}} r\right) .
\end{aligned}
$$

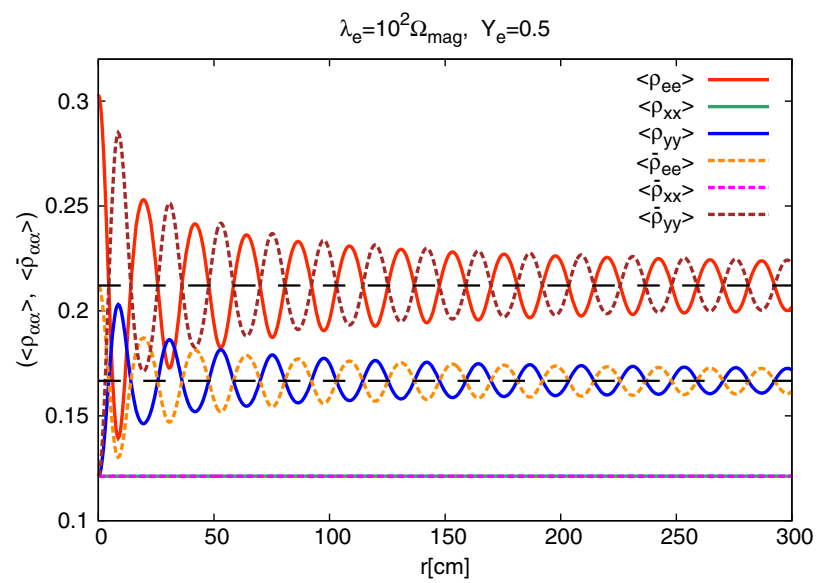

FIG. 5. The evolution of angle averaged diagonal components of neutrino density matrices. We employ the same parameter set as that in the case of $Y_{e}=0.5$ in Fig. 2. The constant values of the black dash lines correspond to equilibrium values of the two flavor neutrino-antineutrino oscillations such as $\left(n_{\nu_{e}}+n_{\nu_{x}}\right) /$ $2 n_{n_{\nu}}=7 / 33$ and $\left(n_{\bar{\nu}_{e}}+n_{\nu_{x}}\right) / 2 n_{n_{\nu}}=1 / 6$.

The $\rho_{x x}$ is constant, and the $\rho_{e e}$ oscillates around $\left(n_{\nu_{e}}+\right.$ $\left.n_{\nu_{x}}\right) / 2 n_{n_{\nu}}$ in this analytical model. Our derivation can be easily applied to the $\bar{\nu}_{e}-\bar{\nu}_{x}-\nu_{y}$ sector by solving evolutions of $X_{y e, i}$ and $X_{y x, i}$ instead of $X_{e y, i}$ and $X_{x y, i}$. We can show that flavor conversions between $\bar{\nu}_{e}$ and $\nu_{y}$ occur around an equilibrium value $\left(n_{\bar{\nu}_{e}}+n_{\nu_{x}}\right) / 2 n_{n_{\nu}}$ and $\bar{\nu}_{x}$ decouples from flavor conversions. The above analytical discussion reproduces numerical results of Fig. 5 qualitatively. Figure 5 represents evolution of angle averaged diagonal components of neutrino density matrices as defined in Eqs. (12) and (13) in the $e-x-y$ basis. The input parameters used in Fig. 5 are the same as those in the case of $Y_{e}=0.5$ in Fig. 2 (blue solid line). Figure 5 shows that one of the flavors $x$ is decoupled from neutrino oscillations and two flavor neutrino-antineutrino oscillations in $\nu_{e}-\bar{\nu}_{y}$ and $\nu_{y}-\bar{\nu}_{e}$ sectors happen around $\left(n_{\nu_{e}}+n_{\nu_{x}}\right) / 2 n_{n_{\nu}}$ and $\left(n_{\bar{\nu}_{e}}+n_{\nu_{x}}\right) / 2 n_{n_{\nu}}$, respectively. The decreasing oscillation amplitudes of $\left\langle\rho_{\alpha \alpha}\right\rangle$ and $\left\langle\bar{\rho}_{\alpha \alpha}\right\rangle$ $(\alpha=e, y)$ up to $r \sim 100 \mathrm{~cm}$ would originate from the angle average on neutrino density matrices.

\section{Case of $\Omega_{\mathrm{mag}} \gg\left|\lambda_{e}-\lambda_{n}\right|, \lambda_{n}$}

The matter potentials are smaller than the magnetic field potential, so the contribution from $X_{x y, i}$ is no longer negligible and $\nu_{x}$ joins flavor conversions. In this case, the second derivatives of $X_{e y, i}$ and $X_{x y, i}$ are written as

$$
\begin{aligned}
& \partial_{r}^{2} X_{e y, i} \sim-4 \Omega_{\mathrm{mag}}^{2} X_{e y, i}-2 \Omega_{\mathrm{mag}}^{2} X_{x y, i}, \\
& \partial_{r}^{2} X_{x y, i} \sim-2 \Omega_{\mathrm{mag}}^{2} X_{e y, i}-4 \Omega_{\mathrm{mag}}^{2} X_{x y, i} .
\end{aligned}
$$

The above differential equations can be solved analytically, and coefficients of two modes, $\sin \left(\sqrt{2} \Omega_{\mathrm{mag}} r\right)$ and $\sin \left(\sqrt{6} \Omega_{\mathrm{mag}} r\right)$, are determined by imposing the initial 


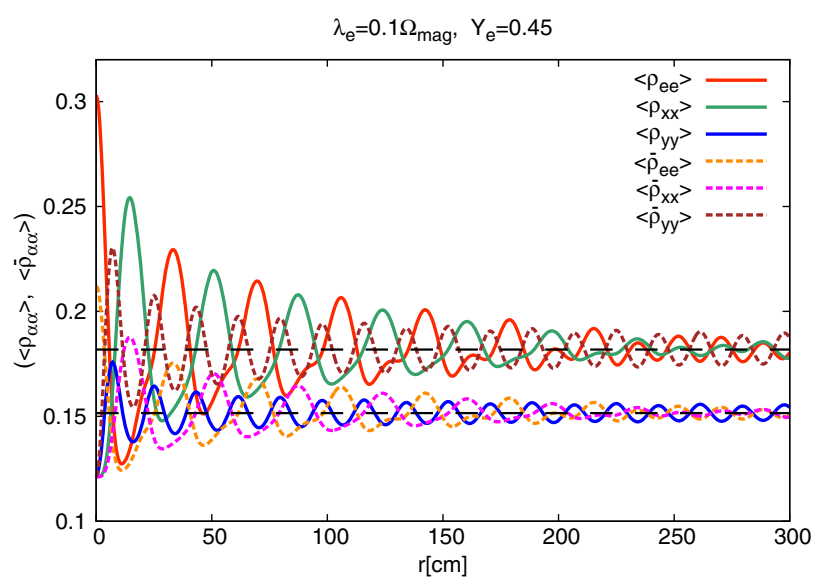

FIG. 6. The evolution of diagonal components like Fig. 5. The input parameters are the same as that in the case of $\lambda_{e}=0.1 \Omega_{\mathrm{mag}}$ in Fig. 1. The constant black dashed lines show equilibrium values of the three flavor neutrino-antineutrino oscillations such as $\left(n_{\nu_{e}}+2 n_{\nu_{x}}\right) / 3 n_{\nu}=2 / 11$ and $\left(n_{\bar{\nu}_{e}}+2 n_{\nu_{x}}\right) / 3 n_{\nu}=5 / 33$.

condition of neutrino density matrices in Eqs. (A16) and (A18). The diagonal terms of neutrino density matrices are given by

$$
\begin{aligned}
\rho_{e e}= & \frac{n_{\nu_{e}}}{n_{\nu}}+\frac{n_{\nu_{x}}-n_{\nu_{e}}}{6 n_{\nu}}\left(1-\cos \sqrt{6} \Omega_{\mathrm{mag}} r\right) \\
& +\frac{n_{\nu_{x}}-n_{\nu_{e}}}{2 n_{\nu}}\left(1-\cos \sqrt{2} \Omega_{\mathrm{mag}} r\right), \\
\rho_{x x}= & \frac{n_{\nu_{x}}}{n_{\nu}}+\frac{n_{\nu_{x}}-n_{\nu_{e}}}{6 n_{\nu}}\left(1-\cos \sqrt{6} \Omega_{\mathrm{mag}} r\right) \\
& -\frac{n_{\nu_{x}}-n_{\nu_{e}}}{2 n_{\nu}}\left(1-\cos \sqrt{2} \Omega_{\mathrm{mag}} r\right), \\
\bar{\rho}_{y y}= & \frac{n_{\nu_{x}}}{n_{\nu}}-\frac{n_{\nu_{x}}-n_{\nu_{e}}}{3 n_{\nu}}\left(1-\cos \sqrt{6} \Omega_{\mathrm{mag}} r\right) .
\end{aligned}
$$

These diagonal components oscillate around $\left(n_{\nu_{e}}+2 n_{\nu_{x}}\right) /$ $3 n_{\nu}$. Such an analytical model helps us understand numerical results of three flavor oscillations as shown in our simulations [e.g., $\lambda_{e}=0.1 \Omega_{\mathrm{mag}}$ (red solid line) in the top panel of Fig. 1]. In the $\bar{\nu}_{e}-\bar{\nu}_{x}-\nu_{y}$ sector, three flavor oscillations around $\left(n_{\bar{\nu}_{e}}+2 n_{\nu_{x}}\right) / 3 n_{\nu}$ are obtained analytically in the same way as the above derivation. Figure 6 shows numerical results of the three flavor neutrinoantineutrino oscillations. There are the three flavor neutrino-antineutrino oscillations in both $\nu_{e}-\nu_{x}-\bar{\nu}_{y}$ and $\bar{\nu}_{e}-$ $\bar{\nu}_{x}-\nu_{y}$ sectors as discussed in our analytical treatment. The equilibrium values of flavor conversions in Fig. 6 are different from that in Fig. 5 because of the finite couplings of $\nu_{x}$ and $\bar{\nu}_{x}$ with the neutrino-antineutrino oscillations.

\section{APPENDIX B: NEUTRINO POTENTIALS IN AN ELECTRON CAPTURE SUPERNOVA}

This section shows how strong the magnetic field must be to induce the flavor equilibration in ECSN, which has an
$\mathrm{O}-\mathrm{Ne}-\mathrm{Mg}$ core in the progenitor. This section is parallel to Sec. III B, in which we discuss the iron core progenitor. We employ the supernova model with an $8.8 M_{\odot}$ progenitor, which is the same as that of Ref. [47].

In the early explosion phase at $31 \mathrm{~ms}$ postbounce (the top panel of Fig. 7), there is a sharp drop of $\lambda$ around $r=100 \mathrm{~km}$, which corresponds to the position of the propagating shock wave. The baryon density decreases significantly outside the shock front. The shock wave has reached a layer of $\alpha$ elements where the electron fraction is $Y_{e} \sim 0.5$. The matter potential $\lambda \propto\left|2 Y_{e}-1\right|$ disappears, and neutrino-neutrino interactions become dominant in such a region, and $\eta$ is equivalent to $\zeta$. The enhancement of the $\eta$ at $r=838 \mathrm{~km}$ would reflect the large electron fraction of $\mathrm{He}+\mathrm{H}$ layers in our progenitor model. The value of $\lambda$ is sensitive to composition of nuclear species in supernova material because of the $Y_{e}$ dependence. Therefore, the radial profile of $\lambda$ as shown in the top panel of Fig. 7 would be a unique structure of ECSN which has an $\mathrm{O}-\mathrm{Mg}-\mathrm{Ne}$ core in the progenitor. In the outer region $(r>2120 \mathrm{~km}), \eta=\omega$ is satisfied because of the decreasing baryon density and neutrino fluxes. The value of $\eta$ (blue solid line) is always larger than that of $\Omega_{\text {mag }}$ (magenta solid
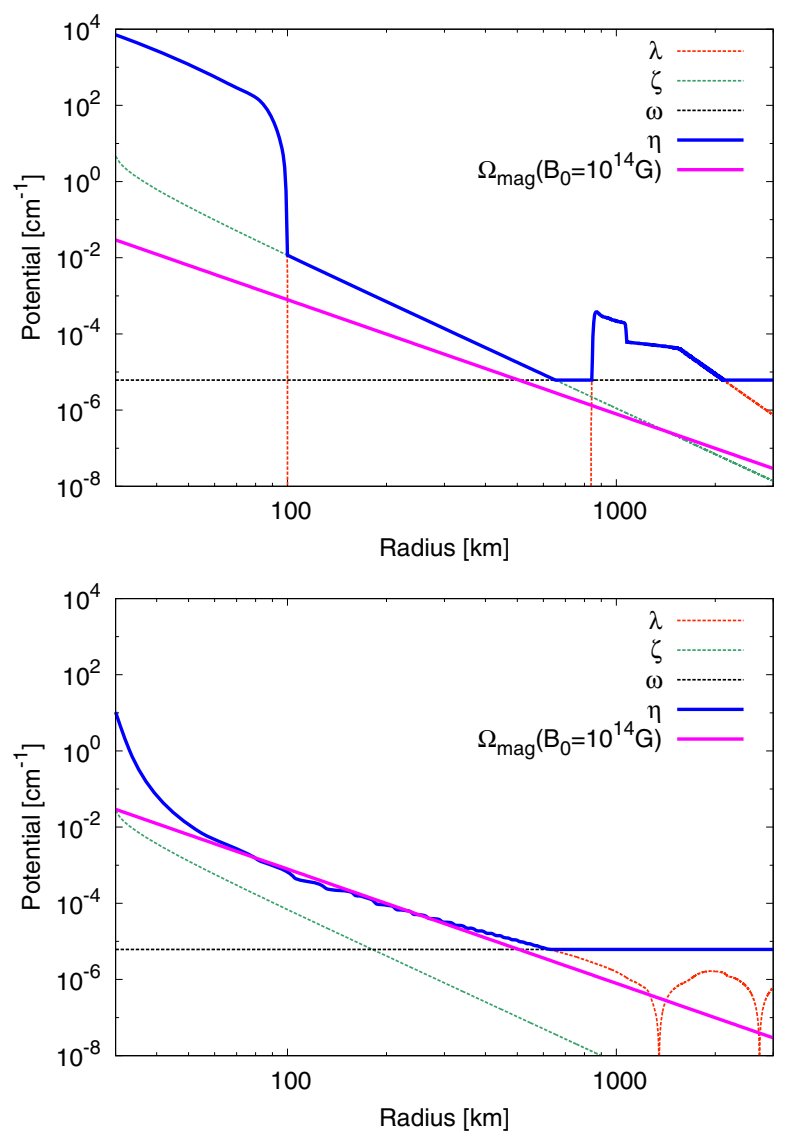

FIG. 7. The radial profiles of neutrino potentials in ECSN as in Fig. 3. The top (bottom) panel shows the case at 31 (331) ms postbounce. 


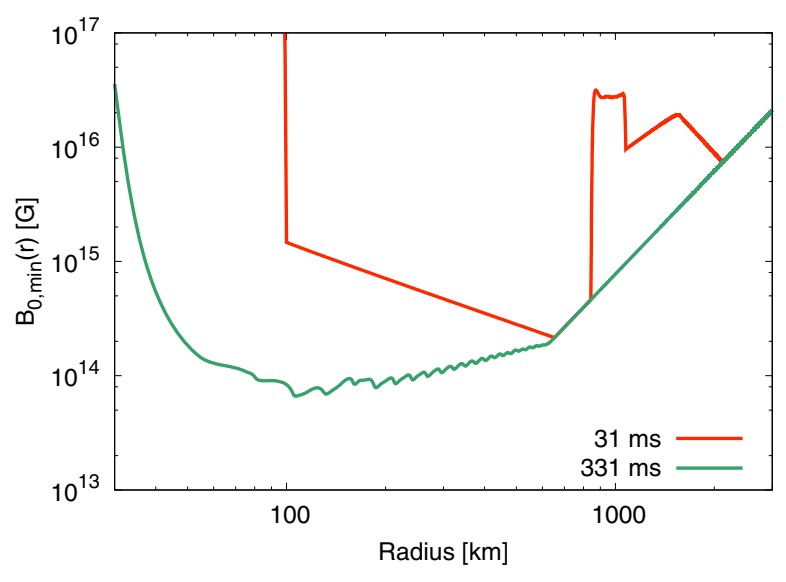

FIG. 8. Radial profiles of Eq. (18) at 31 and $331 \mathrm{~ms}$ postbounce in ECSN.

line), so $B_{0}=10^{14} \mathrm{G}$ is not enough to satisfy Eq. (17) at $31 \mathrm{~ms}$ postbounce.

The radial profiles of potentials at $331 \mathrm{~ms}$ postbounce are shown in the bottom panel of Fig. 7. The potential $\zeta$ is not a dominant term in the neutrino Hamiltonian because the neutrino luminosity on the surface of the PNS decreases as the explosion time has passed. Therefore, in the later explosion phase, the contribution from neutrino-neutrino interactions to the neutrino-antineutrino oscillations is negligible. On the other hand, the matter effect becomes dominant up to $r=631 \mathrm{~km}$. The shock wave propagates outward, heating material and changing the value of $Y_{e}$ in the outer layers of the progenitor model. The vacuum potential $\omega$ is the largest term among neutrino potentials in outer region $(r>631 \mathrm{~km})$. The decreasing baryon density near the surface of the PNS reduces the value of $\lambda$, which results in the crossing of $\eta$ (blue solid line) and $\Omega_{\text {mag }}$ (magenta solid line) in the bottom panel of Fig. 7.

Figure 8 represents the radial profiles of Eq. (18) in the ECSN. The minimum value of $B_{0, \min }(r)$ at 31 (331) ms postbounce is $2.16 \times 10^{14}\left(6.62 \times 10^{13}\right) \mathrm{G}$. In the early explosion phase (red solid line), the decreasing $B_{0, \min }(r)$ at $r=100-838 \mathrm{~km}$ reflects decreasing $\zeta$, so neutrinoneutrino interactions suppress the neutrino-antineutrino oscillations even though matter potential $\lambda$ disappears at the outer layer of $\alpha$ elements. The dilute baryon density in the later explosion phase induces small values of $B_{0, \min }(r)$ around $100 \mathrm{~km}$ (green solid line). In a core-collapse scenario, to produce a magnetar whose magnetic field is approximately $10^{14} \mathrm{G}$, the equilibration of neutrinoantineutrino oscillations would be possible in the later explosion phase.
[1] E. Vitagliano, I. Tamborra, and G. Raffelt, Grand unified neutrino spectrum at earth: Sources and spectral components, Rev. Mod. Phys. 92, 045006 (2020).

[2] K. Hirata et al. (Kamiokande-II Collaboration), Observation of a Neutrino Burst from the Supernova SN 1987a, Phys. Rev. Lett. 58, 1490 (1987).

[3] R. M. Bionta et al., Observation of a Neutrino Burst in Coincidence with Supernova SN 1987a in the Large Magellanic Cloud, Phys. Rev. Lett. 58, 1494 (1987).

[4] E. N. Alekseev, L. N. Alekseeva, I. V. Krivosheina, and V. I. Volchenko, Detection of the neutrino signal from SN1987A in the LMC using the INR baksan underground scintillation telescope, Phys. Lett. B 205, 209 (1988).

[5] S. Horiuchi and J. P. Kneller, What can be learned from a future supernova neutrino detection?, J. Phys. G 45, 043002 (2018).

[6] H.-T. Janka, Neutrino Emission from Supernovae, in Handbook of Supernovae, 1966 (Springer International, Cham, 2017), pp. 1575-1604.

[7] W. Hix, E. Lentz, S. Bruenn, A. Mezzacappa, O. Messer, E. Endeve, J. Blondin, J. Harris, P. Marronetti, and K. Yakunin, The multi-dimensional character of core-collapse supernovae, Acta Phys. Pol. B 47, 645 (2016).

[8] B. Müller, The status of multi-dimensional core-collapse supernova models, Pub. Astron. Soc. Aust. 33, e048 (2016).
[9] A. Mirizzi, I. Tamborra, H.-T. Janka, N. Saviano, K. Scholberg, R. Bollig, L. Hudepohl, and S. Chakraborty, Supernova neutrinos: Production, oscillations and detection, Riv. Nuovo Cimento 39, 1 (2015).

[10] T. Foglizzo, R. Kazeroni, J. Guilet, F. Masset, M. González, B. K. Krueger, J. Novak, M. Oertel, J. Margueron, J. Faure, N. Martin, P. Blottiau, B. Peres, and G. Durand, The explosion mechanism of core-collapse supernovae: Progress in supernova theory and experiments, Pub. Astron. Soc. Aust. 32, e009 (2015).

[11] A. Burrows, Colloquium: Perspectives on core-collapse supernova theory, Rev. Mod. Phys. 85, 245 (2013).

[12] K. Kotake, K. Sumiyoshi, S. Yamada, T. Takiwaki, T. Kuroda, Y. Suwa, and H. Nagakura, Core-collapse supernovae as supercomputing science: A status report toward six-dimensional simulations with exact Boltzmann neutrino transport in full general relativity, Prog. Theor. Exp. Phys. 2012, 01A301 (2012).

[13] L. Wolfenstein, Neutrino oscillations in matter, Phys. Rev. D 17, 2369 (1978).

[14] S. P. Mikheyev and A. Yu. Smirnov, Resonance amplification of oscillations in matter and spectroscopy of solar neutrinos, Sov. J. Nucl. Phys. 42, 913 (1985).

[15] G. M. Fuller, R. W. Mayle, J. R. Wilson, and D. N. Schramm, Resonant neutrino oscillations and stellar collapse, Astrophys. J. 322, 795 (1987). 
[16] J. T. Pantaleone, Neutrino oscillations at high densities, Phys. Lett. B 287, 128 (1992).

[17] J. T. Pantaleone, Dirac neutrinos in dense matter, Phys. Rev. D 46, 510 (1992).

[18] G. Sigl and G. Raffelt, General kinetic description of relativistic mixed neutrinos, Nucl. Phys. B406, 423 (1993).

[19] B. H. J. McKellar and M. J. Thomson, Oscillating doublet neutrinos in the early universe, Phys. Rev. D 49, 2710 (1994).

[20] S. Yamada, Boltzmann equations for neutrinos with flavor mixings, Phys. Rev. D 62, 093026 (2000).

[21] A. B. Balantekin and Y. Pehlivan, Neutrino-neutrino interactions and flavor mixing in dense matter, J. Phys. G 34, 47 (2007).

[22] C. Y. Cardall, Liouville equations for neutrino distribution matrices, Phys. Rev. D 78, 085017 (2008).

[23] Y. Pehlivan, A. B. Balantekin, T. Kajino, and T. Yoshida, Invariants of collective neutrino oscillations, Phys. Rev. D 84, 065008 (2011).

[24] A. Vlasenko, G. M. Fuller, and V. Cirigliano, Neutrino quantum kinetics, Phys. Rev. D 89, 105004 (2014).

[25] C. Volpe, D. Vnnen, and C. Espinoza, Extended evolution equations for neutrino propagation in astrophysical and cosmological environments, Phys. Rev. D 87, 113010 (2013).

[26] D. N. Blaschke and V. Cirigliano, Neutrino quantum kinetic equations: The collision term, Phys. Rev. D 94, 033009 (2016).

[27] S. Birol, Y. Pehlivan, A. B. Balantekin, and T. Kajino, Neutrino spectral split in the exact many body formalism, Phys. Rev. D 98, 083002 (2018).

[28] S. A. Richers, G. C. McLaughlin, J. P. Kneller, and A. Vlasenko, Neutrino quantum kinetics in compact objects, Phys. Rev. D 99, 123014 (2019).

[29] H. Duan and J. P. Kneller, Neutrino flavour transformation in supernovae, J. Phys. G 36, 113201 (2009).

[30] H. Duan, G. M. Fuller, J. Carlson, and Y.-Z. Qian, Coherent Development of Neutrino Flavor in the Supernova Environment, Phys. Rev. Lett. 97, 241101 (2006).

[31] H. Duan, G. M. Fuller, J. Carlson, and Y.-Z. Qian, Simulation of coherent non-linear neutrino flavor transformation in the supernova environment. 1. correlated neutrino trajectories, Phys. Rev. D 74, 105014 (2006).

[32] G. L. Fogli, E. Lisi, A. Marrone, and A. Mirizzi, Collective neutrino flavor transitions in supernovae and the role of trajectory averaging, J. Cosmol. Astropart. Phys. 12 (2007) 010 .

[33] G. G. Raffelt and A. Yu. Smirnov, Adiabaticity and spectral splits in collective neutrino transformations, Phys. Rev. D 76, 125008 (2007).

[34] B. Dasgupta and A. Dighe, Collective three-flavor oscillations of supernova neutrinos, Phys. Rev. D 77, 113002 (2008).

[35] B. Dasgupta, A. Dighe, G. G. Raffelt, and A. Yu. Smirnov, Multiple Spectral Splits of Supernova Neutrinos, Phys. Rev. Lett. 103, 051105 (2009).

[36] B. Dasgupta, A. Mirizzi, I. Tamborra, and R. Tomas, Neutrino mass hierarchy and three-flavor spectral splits of supernova neutrinos, Phys. Rev. D 81, 093008 (2010).
[37] H. Duan and A. Friedland, Self-Induced Suppression of Collective Neutrino Oscillations in a Supernova, Phys. Rev. Lett. 106, 091101 (2011).

[38] A. Friedland, Self-Refraction of Supernova Neutrinos: Mixed Spectra and Three-Flavor Instabilities, Phys. Rev. Lett. 104, 191102 (2010).

[39] A. Mirizzi and R. Tomas, Multi-angle effects in selfinduced oscillations for different supernova neutrino fluxes, Phys. Rev. D 84, 033013 (2011).

[40] J. F. Cherry, G. M. Fuller, J. Carlson, H. Duan, and Y.-Z. Qian, Multi-angle simulation of flavor evolution in the neutrino neutronization burst from an O-Ne-Mg core-collapse supernova, Phys. Rev. D 82, 085025 (2010).

[41] J. F. Cherry, M.-R. Wu, J. Carlson, H. Duan, G. M. Fuller, and Y.-Z. Qian, Density fluctuation effects on collective neutrino oscillations in $\mathrm{O}-\mathrm{Ne}-\mathrm{Mg}$ core-collapse supernovae, Phys. Rev. D 84, 105034 (2011).

[42] S. Chakraborty, T. Fischer, A. Mirizzi, N. Saviano, and R. Tomas, No Collective Neutrino Flavor Conversions During The Supernova Accretion Phase, Phys. Rev. Lett. 107, 151101 (2011).

[43] S. Chakraborty, T. Fischer, A. Mirizzi, N. Saviano, and R. Tomas, Analysis of matter suppression in collective neutrino oscillations during the supernova accretion phase, Phys. Rev. D 84, 025002 (2011).

[44] M.-R. Wu, Y.-Z. Qian, G. Martinez-Pinedo, T. Fischer, and L. Huther, Effects of neutrino oscillations on nucleosynthesis and neutrino signals for an $18 \mathrm{M}$ supernova model, Phys. Rev. D 91, 065016 (2015).

[45] H. Sasaki, T. Kajino, T. Takiwaki, T. Hayakawa, A. B. Balantekin, and Y. Pehlivan, Possible effects of collective neutrino oscillations in three-flavor multiangle simulations of supernova $\nu p$ processes, Phys. Rev. D 96, 043013 (2017).

[46] M. Zaizen, T. Yoshida, K. Sumiyoshi, and H. Umeda, Collective neutrino oscillations and detectabilities in failed supernovae, Phys. Rev. D 98, 103020 (2018).

[47] H. Sasaki, T. Takiwaki, S. Kawagoe, S. Horiuchi, and K. Ishidoshiro, Detectability of collective neutrino oscillation signatures in the supernova explosion of a $8.8 M_{\odot}$ star, Phys. Rev. D 101, 063027 (2020).

[48] M. Zaizen, J. F. Cherry, T. Takiwaki, S. Horiuchi, K. Kotake, H. Umeda, and T. Yoshida, Neutrino halo effect on collective neutrino oscillation in iron core-collapse supernova model of a $9.6 \mathrm{M}_{\text {solar }}$ star, J. Cosmol. Astropart. Phys. 06 (2020) 011.

[49] M. Zaizen, S. Horiuchi, T. Takiwaki, K. Kotake, T. Yoshida, H. Umeda, and J. F. Cherry, Three-flavor collective neutrino conversions with multi-azimuthal-angle instability in an electron-capture supernova model, Phys. Rev. D 103, 063008 (2021).

[50] A. Esteban-Pretel, A. Mirizzi, S. Pastor, R. Tomas, G. G. Raffelt, P. D. Serpico, and G. Sigl, Role of dense matter in collective supernova neutrino transformations, Phys. Rev. D 78, 085012 (2008).

[51] N. Saviano, S. Chakraborty, T. Fischer, and A. Mirizzi, Stability analysis of collective neutrino oscillations in the supernova accretion phase with realistic energy and angle distributions, Phys. Rev. D 85, 113002 (2012). 
[52] G. Martinez-Pinedo, B. Ziebarth, T. Fischer, and K. Langanke, Effect of collective neutrino flavor oscillations on vp-process nucleosynthesis, Eur. Phys. J. A 47, 98 (2011).

[53] E. Pllumbi, I. Tamborra, S. Wanajo, H.-T. Janka, and L. Hüdepohl, Impact of neutrino flavor oscillations on the neutrino-driven wind nucleosynthesis of an electron-capture supernova, Astrophys. J. 808, 188 (2015).

[54] Z. Xiong, A. Sieverding, M. Sen, and Y.-Z. Qian, Potential impact of fast flavor oscillations on neutrino-driven winds and their nucleosynthesis, Astrophys. J. 900, 144 (2020).

[55] H. Ko et al., Neutrino process in core-collapse supernovae with neutrino self-interaction and MSW effects, Astrophys. J. Lett. 891, L24 (2020).

[56] A. Malkus, A. Friedland, and G. C. McLaughlin, Matterneutrino resonance above merging compact objects, arXiv:1403.5797.

[57] A. Malkus, G. C. McLaughlin, and R. Surman, Symmetric and standard matter-neutrino resonances above merging compact objects, Phys. Rev. D 93, 045021 (2016).

[58] M.-R. Wu, H. Duan, and Y.-Z. Qian, Physics of neutrino flavor transformation through matter-neutrino resonances, Phys. Lett. B 752, 89 (2016).

[59] Y.-L. Zhu, A. Perego, and G. C. McLaughlin, Matter neutrino resonance transitions above a neutron star merger remnant, Phys. Rev. D 94, 105006 (2016).

[60] M. Frensel, M.-R. Wu, C. Volpe, and A. Perego, Neutrino flavor evolution in binary neutron star merger remnants, Phys. Rev. D 95, 023011 (2017).

[61] A. Chatelain and C. Volpe, Helicity coherence in binary neutron star mergers and non-linear feedback, Phys. Rev. D 95, 043005 (2017).

[62] J. Y. Tian, A. V. Patwardhan, and G. M. Fuller, Neutrino flavor evolution in neutron star mergers, Phys. Rev. D 96, 043001 (2017).

[63] A. Vlasenko and G. C. McLaughlin, Matter-neutrino resonance in a multiangle neutrino bulb model, Phys. Rev. D 97, 083011 (2018).

[64] S. Shalgar, Multi-angle calculation of the matter-neutrino resonance near an accretion disk, J. Cosmol. Astropart. Phys. 02 (2018) 010.

[65] M.-R. Wu, I. Tamborra, O. Just, and H.-T. Janka, Imprints of neutrino-pair flavor conversions on nucleosynthesis in ejecta from neutron-star merger remnants, Phys. Rev. D 96, 123015 (2017).

[66] M. George, M.-R. Wu, I. Tamborra, R. Ardevol-Pulpillo, and H.-T. Janka, Fast neutrino flavor conversion, ejecta properties, and nucleosynthesis in newly-formed hypermassive remnants of neutron-star mergers, Phys. Rev. D 102, 103015 (2020).

[67] I. Tamborra and S. Shalgar, New developments in flavor evolution of a dense neutrino gas, arXiv:2011.01948 [Ann. Rev. Nucl. Part. Sci. (to be published)].

[68] A. B. Balantekin and B. Kayser, On the properties of neutrinos, Annu. Rev. Nucl. Part. Sci. 68, 313 (2018).

[69] C. Giunti and A. Studenikin, Neutrino electromagnetic interactions: A window to new physics, Rev. Mod. Phys. 87, 531 (2015).

[70] A. Beda, V. Brudanin, V. Egorov, D. Medvedev, V. Pogosov, E. Shevchik, M. Shirchenko, A. Starostin, and
I. Zhitnikov, Gemma experiment: The results of neutrino magnetic moment search, Phys. Part. Nucl. Lett. 10, 139 (2013).

[71] M. Agostini et al. (Borexino Collaboration), Limiting neutrino magnetic moments with Borexino Phase-II solar neutrino data, Phys. Rev. D 96, 091103 (2017).

[72] K. Abe et al. (XMASS Collaboration), Search for exotic neutrino-electron interactions using solar neutrinos in XMASS-I, Phys. Lett. B 809, 135741 (2020).

[73] E. Aprile et al. (XENON Collaboration), Excess electronic recoil events in XENON1T, Phys. Rev. D 102, 072004 (2020).

[74] S. Arceo-Díaz, K. P. Schröder, K. Zuber, and D. Jack, Constraint on the magnetic dipole moment of neutrinos by the tip-RGB luminosity in $\omega$-Centauri, Astropart. Phys. 70, 1 (2015).

[75] F. Capozzi and G. Raffelt, Axion and neutrino bounds improved with new calibrations of the tip of the red-giant branch using geometric distance determinations, Phys. Rev. D 102, 083007 (2020).

[76] K. Mori, A. B. Balantekin, T. Kajino, and M. A. Famiano, Elimination of the blue loops in the evolution of intermediate-mass stars by the neutrino magnetic moment and large extra dimensions, Astrophys. J. 901, 115 (2020).

[77] C.-S. Lim and W. J. Marciano, Resonant spin-flavor precession of solar and supernova neutrinos, Phys. Rev. D 37, 1368 (1988).

[78] E. K. Akhmedov and Z.G. Berezhiani, Implications of Majorana neutrino transition magnetic moments for neutrino signals from supernovae, Nucl. Phys. B373, 479 (1992).

[79] E. K. Akhmedov, S. T. Petcov, and A. Y. Smirnov, Neutrinos with mixing in twisting magnetic fields, Phys. Rev. D 48, 2167 (1993).

[80] T. Totani and K. Sato, Resonant spin flavor conversion of supernova neutrinos and deformation of the anti-electronneutrino spectrum, Phys. Rev. D 54, 5975 (1996).

[81] H. Nunokawa, Y.Z. Qian, and G. M. Fuller, Resonant neutrino spin flavor precession and supernova nucleosynthesis and dynamics, Phys. Rev. D 55, 3265 (1997).

[82] S. Ando and K. Sato, Three generation study of neutrino spin flavor conversion in supernova and implication for neutrino magnetic moment, Phys. Rev. D 67, 023004 (2003).

[83] S. Ando and K. Sato, Resonant spin flavor conversion of supernova neutrinos: Dependence on presupernova models and future prospects, Phys. Rev. D 68, 023003 (2003).

[84] S. Ando and K. Sato, A Comprehensive study of neutrino spin flavor conversion in supernovae and the neutrino mass hierarchy, J. Cosmol. Astropart. Phys. 10 (2003) 001.

[85] E. K. Akhmedov and T. Fukuyama, Supernova prompt neutronization neutrinos and neutrino magnetic moments, J. Cosmol. Astropart. Phys. 12 (2003) 007.

[86] A. Ahriche and J. Mimouni, Supernova neutrino spectrum with matter and spin flavor precession effects, J. Cosmol. Astropart. Phys. 11 (2003) 004.

[87] T. Yoshida, A. Takamura, K. Kimura, H. Yokomakura, S. Kawagoe, and T. Kajino, Resonant spin-flavor conversion of supernova neutrinos: Dependence on electron mole fraction, Phys. Rev. D 80, 125032 (2009). 
[88] M. Dvornikov, Evolution of a dense neutrino gas in matter and electromagnetic field, Nucl. Phys. B855, 760 (2012).

[89] A. de Gouvea and S. Shalgar, Effect of transition magnetic moments on collective supernova neutrino oscillations, J. Cosmol. Astropart. Phys. 10 (2012) 027.

[90] A. de Gouvea and S. Shalgar, Transition magnetic moments and collective neutrino oscillations: Three-flavor effects and detectability, J. Cosmol. Astropart. Phys. 04 (2013) 018.

[91] S. Abbar, Collective oscillations of majorana neutrinos in strong magnetic fields and self-induced flavor equilibrium, Phys. Rev. D 101, 103032 (2020).

[92] Z. Yuan, Y.-F. Li, and X. Zhou, Spin flavor spectral splits of supernova neutrino flavor conversions, arXiv:2105 .07928 .

[93] H. Duan and S. Shalgar, Flavor instabilities in the neutrino line model, Phys. Lett. B 747, 139 (2015).

[94] S. Abbar and M. C. Volpe, On fast neutrino flavor conversion modes in the nonlinear regime, Phys. Lett. B 790, 545 (2019).

[95] T. Takiwaki, K. Kotake, and Y. Suwa, Three-dimensional simulations of rapidly rotating core-collapse supernovae: finding a neutrino-powered explosion aided by nonaxisymmetric flows, Mon. Not. R. Astron. Soc. 461, L112 (2016).

[96] J. Matsumoto, T. Takiwaki, K. Kotake, Y. Asahina, and H. R. Takahashi, 2D numerical study for magnetic field dependence of neutrino-driven core-collapse supernova models, Mon. Not. R. Astron. Soc. 499, 4174 (2020).

[97] T. Takiwaki, K. Kotake, and Y. Suwa, A comparison of two- and three-dimensional neutrino-hydrodynamics simulations of core-collapse supernovae, Astrophys. J. 786, 83 (2014).

[98] K. Kotake, T. Takiwaki, T. Fischer, K. Nakamura, and G. Martínez-Pinedo, Impact of neutrino opacities on corecollapse supernova simulations, Astrophys. J. 853, 170 (2018).

[99] E. O'Connor, R. Bollig, A. Burrows, S. Couch, T. Fischer, H.-T. Janka, K. Kotake, E. J. Lentz, M. Liebendörfer, O. E. B. Messer, A. Mezzacappa, T. Takiwaki, and D. Vartanyan, Global comparison of core-collapse supernova simulations in spherical symmetry, J. Phys. G Nucl. Phys. 45, 104001 (2018).

[100] S. E. Woosley, A. Heger, and T. A. Weaver, The evolution and explosion of massive stars, Rev. Mod. Phys. 74, 1015 (2002).

[101] J. F. Cherry, G. M. Fuller, S. Horiuchi, K. Kotake, T. Takiwaki, and T. Fischer, Time of flight and supernova progenitor effects on the neutrino halo, Phys. Rev. D 102, 023022 (2020).

[102] T. Enoto, S. Kisaka, and S. Shibata, Observational diversity of magnetized neutron stars, Rep. Prog. Phys. 82, 106901 (2019). 